\title{
Syntheses and Optical Characterizations of ZnS:Mn Nanocrystals Capped by Polyethylene Oxide Molecules of Varying Molecular Weights
}

\author{
Song-Yi Kim and Cheong-Soo Hwang* \\ Department of Chemistry, Center for Photofunctional Energy Materials (GRRC), Dankook University, \\ Gyonggi 448-701, Korea.*E-mail: cshwang@dankook.ac.kr \\ Received August 11, 2010, Accepted September 27, 2010
}

Key Words: ZnS:Mn nanocrystal, Polyethylene oxide capping, Water-dispersible nanocrystal, Orange emitting nanophosphor

Low-dimensional semiconducting nano-materials have gained significant attention over the past decade. ${ }^{1,2}$ These materials have found a wide range of applications in non-linear optics or electronic devices, and more recently as some bio-imaging or labeling agents due to their unique physical, chemical and optical properties. ${ }^{3-5}$ Among the most important characteristics of these semiconducting nanocrystals is their optical properties which can vary greatly with the size of the nanocrystals, because the corresponding band gap energies differ even between materials of identical chemical composition. ${ }^{6,7}$

$\mathrm{ZnS}: \mathrm{Mn}$ nanocrystals (an orange light emitting nanophosphor) have attracted substantial interest on account of their high quantum yields and stability at ambient temperature, essential properties of commercial electro-luminescence devices. ${ }^{8}$ Considerable progress has been made in the synthetic techniques used to create such nanocrystals including gas, solid, and aqueous solution reactions via the thermal decomposition of organometallic precursors. However, these methods often require high temperatures and pressures and even the use of bio-hazardous substances. ${ }^{9}$

Water-dispersible semiconducting nanocrystals were developed especially for fluorescent labeling agents in living cells as an advanced bio-imaging technique. ${ }^{10-12}$ They are expected to replace complicated or hazardous radioactive detection, and have formed the basis of very sensitive biological assays. In addition, semiconducting nanocrystals are much more efficient, sensitive and stable than the organic dyes currently used. Unfortunately, most highly luminescent semiconductor nanocrystals are grown in non-polar media, making them largely incompatible with any biological system. To date, there are several reports of solubilized hydrophobic nanocrystals in water, created by modifying the crystals' surfaces; ${ }^{13-15}$ however, generating water-dispersible and bio-compatible semiconductor nanocrystals which maintain high quantum efficiency and stability in a biological system still remains a great challenge. Several biocompatible amino acid molecule-capped ZnS:Mn nanocrystals have been synthesized in this laboratory. ${ }^{16,17}$ In addition, more recently, we have synthesized mercaptoacetate (MAA) molecules capped water-dispersible $\mathrm{ZnS}: \mathrm{Mn}$ nanocrystal. The surface capping MAA molecules were characterized by FT-Raman spectroscopy, and the specific coordination mode of the MAA molecule was determined by a DFT calculation. ${ }^{18}$

This paper describes the synthesis of water-dispersible $\mathrm{ZnS}$ : Mn nanocrystals whose surfaces are capped by polyethylene oxide molecules of varying molecular weight. Their physical and optical properties are also investigated. Historically, the abbreviations PEG (polyethylene glycol) and PEO (polyethylene oxide) refer to oligomers or polymers of ethylene oxide molecules. PEG has tended to refer to oligomers and polymers with a molecular mass below $20,000 \mathrm{~g} / \mathrm{mol}$, while PEO to polymers with a molecular mass above that. ${ }^{19}$ This article refers to all polyethylene oxide molecules, regardless of molecular weight, as PEO for the sake of clarity. PEO has been reported as a capping agent for $\mathrm{CdSe} / \mathrm{ZnS}$ quantum dots, ${ }^{20}$ with $\mathrm{PEO}$ molecules being chemically linked to oligopeptide molecules and then coordinated to the surfaces of $\mathrm{CdSe} / \mathrm{ZnS}$ quantum dots by substituting the TOPO ( $n$-trioctylphosphine oxide) capping ligand. The quantum dots were prepared in a hydrophobic medium. In this study, however, direct coordination of polyethylene oxide molecules to the surface of $\mathrm{ZnS}: \mathrm{Mn}$ nanocrystals was attempted.

The primary goal of this research is synthesizing water-dispersible ZnS:Mn-PEO nanocrystals suitable for bio-imaging. Nanocrystals containing zinc ions are expected to be much less toxic than those of a cadmium base, such as CdSe and CdS nanocrystals, when used in vivo. Even though $\mathrm{ZnS}$ :Mn nanocrystals are currently mainly applied in electronic devices, rather than bio-labeling, this article aims to demonstrate their stability and suitable as candidates for biological imaging agents.

\section{Experimental Section}

Preparation procedures are similar to previously reported methods of amino acid capped $\mathrm{ZnS}$ nanocrystals synthesis via the formation of zinc (II)-aminoacid coordinated complexes as reactive intermediates in aqueous solution. ${ }^{21}$ A solution of $\mathrm{ZnSO}_{4} \cdot 5 \mathrm{H}_{2} \mathrm{O}(1.44 \mathrm{~g}, 5 \mathrm{mmol})$ in $50 \mathrm{~mL}$ of water was slowly added to a $50 \mathrm{~mL}$ aqueous mixture containing $1.0 \mathrm{mmols}$ of polyethylene oxide molecules at ambient temperature. In another flask, $\mathrm{MnSO}_{4} \cdot \mathrm{H}_{2} \mathrm{O}(0.02 \mathrm{~g}, 0.1 \mathrm{mmol})$ and $\mathrm{Na}_{2} \mathrm{~S}(0.40 \mathrm{~g}, 5 \mathrm{mmol})$ were dissolved in $20 \mathrm{~mL}$ of DI water. The mixture was then transferred to the flask containing the $\mathrm{ZnSO}_{4} / \mathrm{PEO}$ mixture with vigorous stirring. The resulting solution was refluxed for 20 hours. Slow cooling to ambient temperature and an addition of ethanol solution resulted in a yellow-white precipitate at the bottom of the flask. Finally, the obtained solids were separated via centrifuging and decanting the supernatant. These were then dried for 24 hours in a vacuum oven. The obtained solids were 
redissolved in water and were characterized by UV-visible, FTIR and PL spectroscopies.

\section{Results and Discussions}

Figures 1 (a) through (f) present HR-TEM images of ZnS:Mn nanocrystals capped by PEO molecules of different molecular weights. Their measured average particle sizes were $3.2 \mathrm{~nm}$ (ZnS:Mn-PEO-1,500); $3.4 \mathrm{~nm}$ (ZnS:Mn-PEO-4,000); $2.9 \mathrm{~nm}$ (ZnS:Mn-PEO-10,000); $3.2 \mathrm{~nm}$ (ZnS:Mn-PEO-35,000); $2.8 \mathrm{~nm}$ (ZnS:Mn-PEO-100,000); and 3.0 nm (ZnS:Mn-PEO-200,000). For the particle size measurements, approximately 200 particles in the corresponding HR-TEM images were measured to calculate average particle sizes, and the relative standard deviation (RSD) values were in the range from 0.8 to 1.4 , which are provided in Table 1. In addition, the appearance of distinct lattice planes of approximately $3 \AA$ separations in the fringe images suggests that all the solid samples comprised single crystals rather than poly-crystalline aggregates. The same HR-TEM equipment also obtained energy dispersive X-ray spectra (EDXS) to confirm the elemental compositions of the solid ZnS:Mn-PEO nanocrystals. The atomic ratios of $\mathrm{Zn}$ to $\mathrm{S}$ were almost 1:1, and the average doping concentration of manganese (II) ions in the $\mathrm{ZnS}: \mathrm{Mn}-\mathrm{PEO}$ nanocrystals was approximately $0.5 \%$. The concentration of the manganese (II) ion in the ZnS:MnPEO nanocrystal was more precisely determined by inductively coupled plasma-atomic emission spectrometry (ICP-AES). The measurements provided averages of the elemental proportion of $\mathrm{Mn}$ ion against the $\mathrm{ZnS}$ parent crystals as being: $0.3 \%(\mathrm{ZnS}$ : Mn-PEO-1,500); 0.2\% (ZnS:Mn-PEO-4,000); 0.2\% (ZnS:MnPEO-10,000); 0.3\% (ZnS:Mn-PEO-35,000); 0.2\% (ZnS:MnPEO-100,000); and 0.2\% (ZnS:Mn-PEO-200,000). The manganese (II) ion doping concentrations in the $\mathrm{ZnS}: \mathrm{Mn}$ crystals were intended to be close to $1.0 \%$, a previously reported optimum doping for PL efficiency in other ligand-capped $\mathrm{ZnS}: \mathrm{Mn}$ nanocrystals. $^{22}$

Figure 2 shows the solution photoluminescence (PL) spectroscopy data of the ZnS:Mn-PEO nanocrystals. The room temperature solution PL spectra show broad emission peaks at around: 603 nm (ZnS:Mn-PEO-1,500); 602 nm (ZnS:Mn-PEO4,000); 604 nm (ZnS:Mn-PEO-10,000); 603 nm (ZnS:Mn-PEO35,000); 604 nm (ZnS:Mn-PEO-100,000); and 605 nm (ZnS: Mn-PEO-200,000). These emission spectra were obtained by fixing the excitation wavelengths of the light source corresponding to UV-visible absorption wavelengths. In these spectra, all the ZnS:Mn-PEO nanocrystals showed quite large Stokes shifts between the absorption and emission wavelengths of $c a$. $270 \mathrm{~nm}$. Large Stokes shifts are usually the result of metal or counter anion vacancy in the crystal lattice or surface defects resulting from incomplete capping by the surfactant ligand. ${ }^{23}$ For the $\mathrm{ZnS}$ :Mn-PEO nanocrystals, the orange light emitted at $570 \mathrm{~nm}$ is attributed to the ${ }^{4} \mathrm{~T}_{1}-{ }^{6} \mathrm{~A}_{1}$ transition of the $\mathrm{Mn}^{2+}$ ions. During the luminescence pathway, if surface defect states are located close to the conduction band, the direct energy transfer from the $\mathrm{ZnS}$ host to the $\mathrm{Mn}^{2+}$ dopant ion is significantly interrupted, which can cause a weakening of the orange emission as well as enlarging the Stokes shift. ${ }^{24}$ In some cases, previously mentioned surface defects on the $\mathrm{ZnS}: \mathrm{Mn}$ nanocrystal and zinc
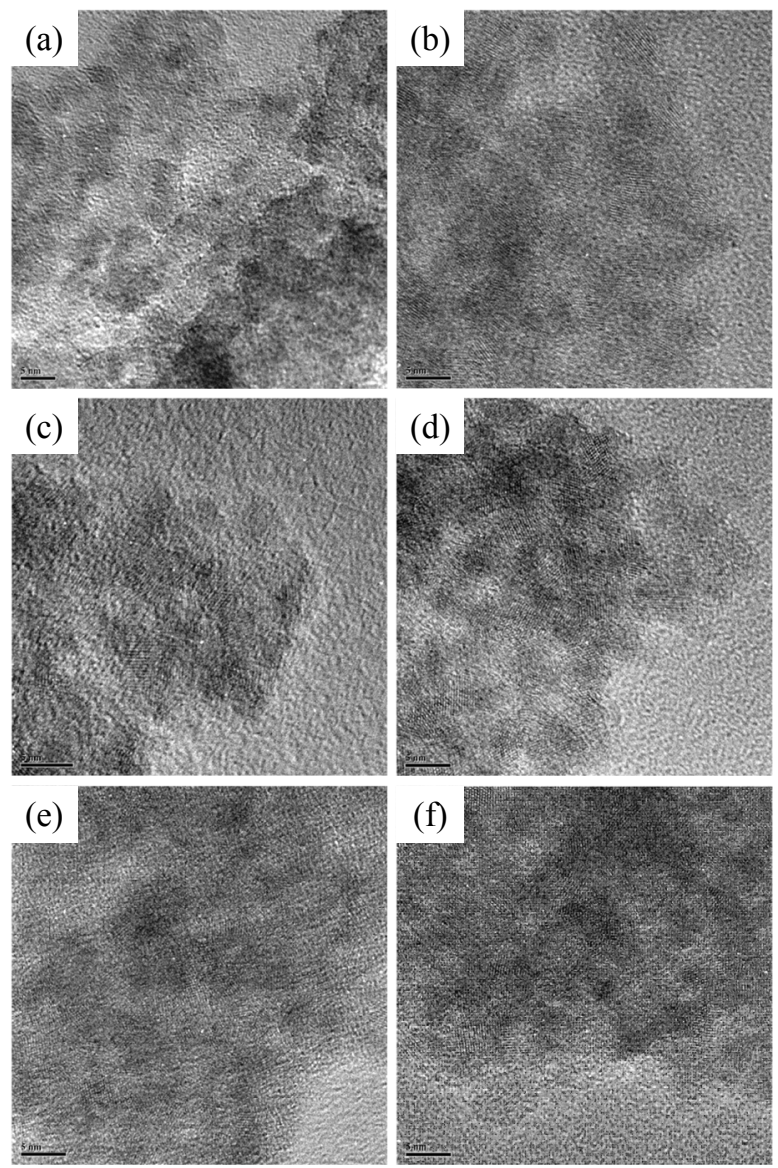

Figure 1. HR-TEM images of: (a) ZnS:Mn-PEO-1,500; (b) ZnS:MnPEO-4,000; (c) ZnS:Mn-PEO-10,000; (d) ZnS:Mn-PEO-35,000; (e) ZnS:Mn-PEO-100,000; and (f) ZnS:Mn-PEO-200,000.

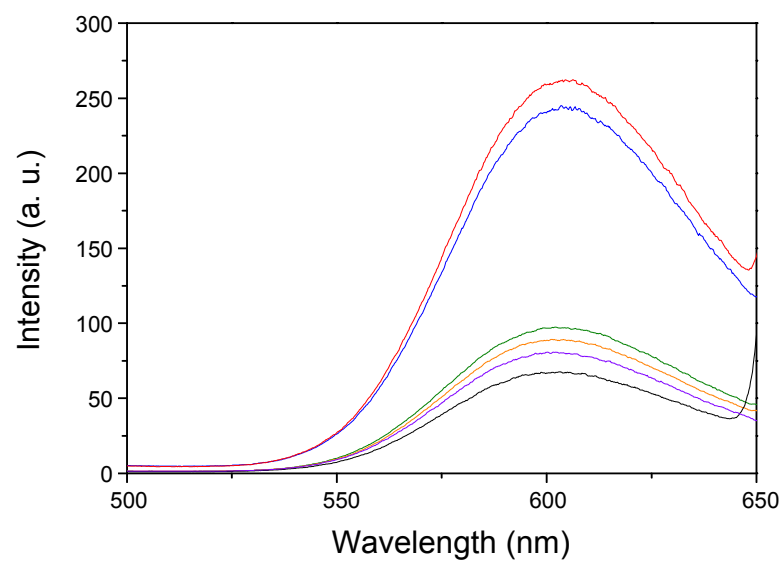

Figure 2. PL emission spectra of: ZnS:Mn-PEO-1,500 (violet); ZnS: Mn-PEO-4,000 (black); ZnS:Mn-PEO-10,000 (blue); ZnS:Mn-PEO35,000 (red); ZnS:Mn-PEO-100,000 (orange); and ZnS:Mn-PEO200,000 (green).

ion vacancies can cause an extra blue emission at around 450 $\mathrm{nm}$, but this was not observed in the PL spectra of these $\mathrm{ZnS}$ : Mn-PEO nanocrystals. ${ }^{25}$

The calculated relative PL efficiencies of the nanocrystals were: 1.9\% (ZnS:Mn-PEO-1,500); 1.8\% (ZnS:Mn-PEO-4,000); 5.3\% (ZnS:Mn-PEO-10,000); 5.9\% (ZnS:Mn-PEO-35,000); 2.5\% (ZnS:Mn-PEO-100,000); and 2.7\% (ZnS:Mn-PEO- 


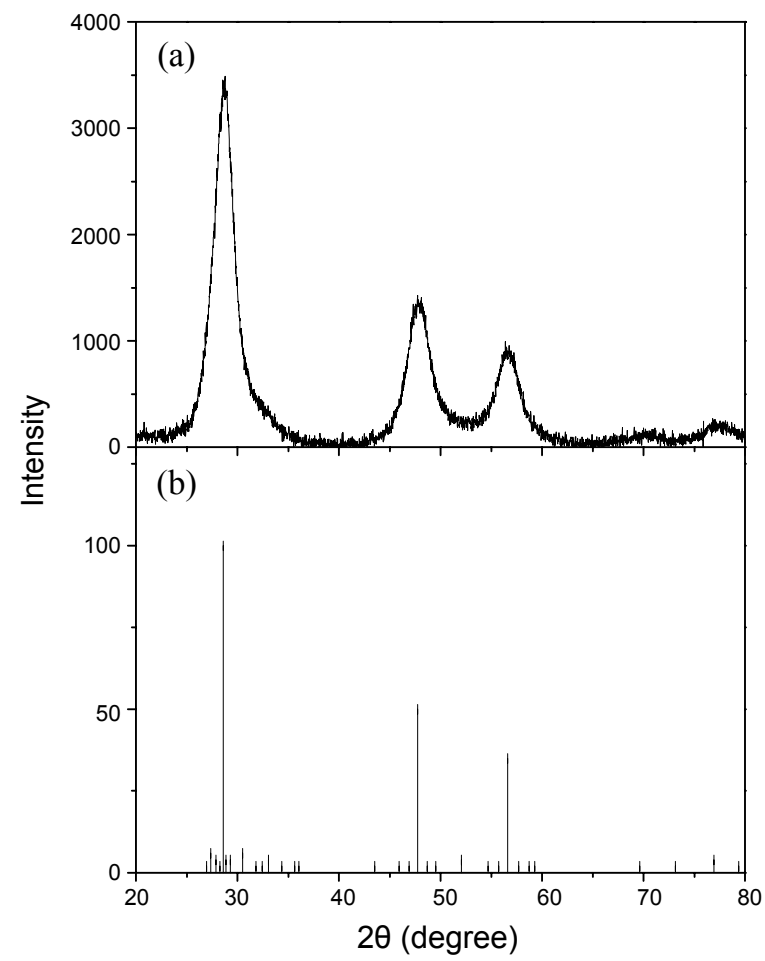

Figure 3. XRD pattern diagram of (a) ZnS:Mn-PEO-35,000 and (b) bulk $\mathrm{ZnS}$ solid in a Wurtzite phase.

200,000). These efficiencies were measured and calculated using a method reported by Williams et al. ${ }^{26}$ which involves calculating relative quantum yields through a comparison with a standard material: a $0.1 \mathrm{M}$ aqueous solution of Tyrosine whose reported excitation wavelength and absolute quantum yield are $275 \mathrm{~nm}$ and 14\%, respectively. ${ }^{27}$ The best PL yield was obtained from the ZnS:Mn-PEO-35,000 nanocrystal, and so it can be concluded this is the most efficient surface capping agent of $\mathrm{ZnS}: \mathrm{Mn}$ nanocrystals dispersed in water. This can be compared to a previous report stating PEO-5,000 as the biologically best capping agent for $\mathrm{CdSe} / \mathrm{ZnS}$ quantum dots. ${ }^{20}$ In addition, the obtained PL efficiency was similar to those for CdSe $(8.9 \%)$, $\mathrm{CdS}(6.0 \%)$, and $\mathrm{ZnS}: \mathrm{Mn}$-Aminoacids $(4.0 \sim 7.0 \%)$ measured in aqueous solutions. ${ }^{17,26}$ Even though the PL efficiency of $\mathrm{ZnS}$ : $\mathrm{Mn}-\mathrm{PEO}$ is lower than that of core-shell type CdSe/ZnS and $\mathrm{ZnS}: \mathrm{Mn} / \mathrm{ZnS}$ nanocrystals, the core-shell quantum dots have some critical disadvantages over the ZnS:Mn-PEO nanocrystal; for instance, their preparation methods are much more complicated and the product yields are usually less than $1 \%$ which is insufficient for any further application study. ${ }^{9}$ It is notable to mention that PEO-10,000 also showed a similar PL efficiency to PEO-35,000, indicating that PEO with molecular weight between 10,000 to 100,000 can be effective surface capping agents for the $\mathrm{ZnS}: \mathrm{Mn}$ nanocrystals whose diameters are about $4 \mathrm{~nm}$. A theoretical calculation suggested that minimum chain length corresponding to PEO-1500 is needed to entirely surround spherical nanoparticle with about $4 \mathrm{~nm}$ diameter. In our result, however, PEO-1500 and PEO-4000 showed relatively low quantum efficiencies, which is indicating that they cannot sufficiently capping the surfaces of the $\mathrm{ZnS}$ :Mn nanocrystal. In these cases, some of the $\mathrm{ZnS}: \mathrm{Mn}$ nanocrystal lattice surfaces are exposed to aqueous solvent molecules which can attack the $\mathrm{Zn}$ ions, resulting them to be dissociated from the crystal lattice to create some $\mathrm{Zn}$ ion vacancies on the surface of the nanocrystal. It is known that cation or anion vacancies can cause significant lowering of the quantum efficiencies for the $\mathrm{ZnS}: \mathrm{Mn}$ nanocrystals. ${ }^{16}$ In addition, PEO-100,000 and PEO-200,000 capped ZnS:Mn nanocrystal also showed relatively low quantum yields. This can be resulted from the fact that those PEO chains are too long for capping the $\mathrm{ZnS}: \mathrm{Mn}$ nanocrystal surface so that the residual chains strongly interact with solvent water molecules, which can also quench the luminescence of the nanocrystal. $^{28}$

The stabilities of colloidal dispersions of the PEO capped nanocrystals were tested under various conditions. First, their dispersibility and photostability in aqueous solutions were tested by storing the colloids, prepared with $c a .5 \mathrm{mg}$ nanocrystal solid dispersed in $10 \mathrm{~mL}$ water, in covered quartz cuvettes under room light exposure for $c a$. 60 days. No precipitation was observed inside the cuvettes during that period, and further PL spectra from the colloids showed that there were no significant changes in the intensities of the emission peaks when compared with the fresh samples of Fig. 2, indicating that there were no significant changes in the PEO capped $\mathrm{ZnS}: \mathrm{Mn}$ nanocrystals, and that they maintain fairly good dispersibility in water for at least 60 days. However, addition of ethanol resulted in the formation of off-white cloudy solutions for both the colloidal solutions. The second stability test for the colloids was their placing in a stainless steel pressure bomb and keeping the colloids under $10 \mathrm{~atm}$ at $120{ }^{\circ} \mathrm{C}$ for $\mathrm{ca} .3$ hours. This test is very important if the colloids were to undergo further preparation for use with sterilized biological samples. The solutions which resulted were clear with no precipitation and no color change; moreover, the obtained PL spectra showed almost identical PL intensities compared with the corresponding original colloidal solutions, indicating that there were no significant changes in the nanocrystals during the test procedures. Finally, the colloidal stability of the ZnS:Mn-PEO nanocrystals were also tested in $0.1 \mathrm{M}$ Tris buffer solutions ( $\mathrm{PH}=7.5$ and 10.0), which are frequently used for cell cultures and bio-analyses. ${ }^{29} \mathrm{We}$ confirmed that the ZnS:Mn-PEO nanocrystals have almost same stability in the Tris buffer solutions as in water.

Figure 3 shows wide angle X-ray diffraction patterns of a powdered sample of PEO-35,000 capped ZnS:Mn nanocrystal (those which afforded the best PL yield) and a bulk $\mathrm{ZnS}$ solid in the Wurtzeite crystalline phase for comparison. In the diagram, most peaks obtained by the ZnS:Mn-PEO-35,000 nanocrystal are broad; however, this is known as a quite common feature of low-dimensional nano-sized semiconducting materials. ${ }^{30}$ Even so, there were obviously indexable peaks such as (008), (110) and (118) planes in the diagram, indicating that the $\mathrm{ZnS}$ : Mn-PEO-35,000 nanocrystal is in a Wurtzite phase with the space group of $P 6_{3} m c{ }^{31}$ All other $\mathrm{ZnS}$ :Mn-PEO nanocrystals showed very similar XRD patterns to the ZnS:Mn-PEO-35,000, which indicate they formed the same parent crystal lattices regardless of capping ligand.

Finally, the capping ligands, polyethylene oxide molecules, were characterized by FT-Raman spectroscopy. Figure 4 and Table 1 present the FT-Raman spectra and identifiable peaks 


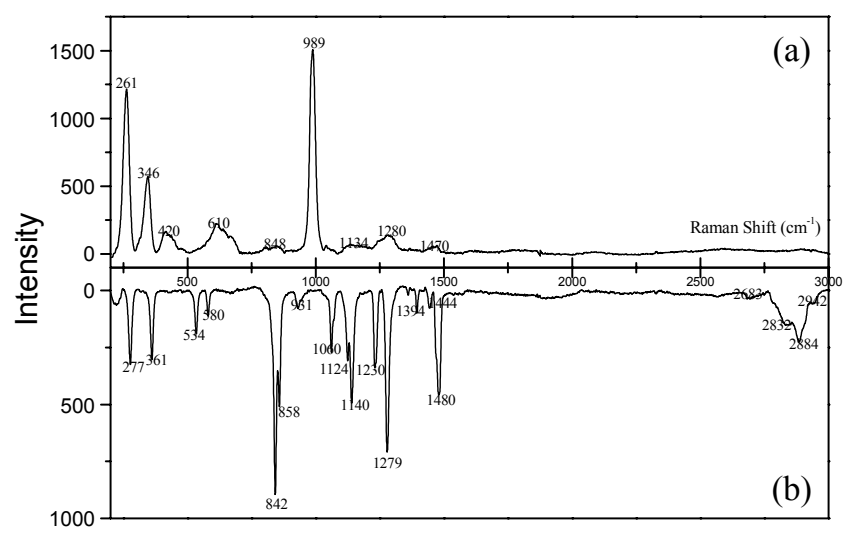

Figure 4. FT-Raman spectra of (a) ZnS:Mn-PEO-35,000 and (B) uncoordinated PEO-35,000 molecule.

Table 1. FT-Raman frequencies of ZnS:Mn-PEO-35,000 and uncoordinated PEO-35,000

\begin{tabular}{ccc}
\hline ZnS:Mn-PEO-35,000 & PEO-35,000 & Assignments $^{29}$ \\
\hline $261(\mathrm{~s})$ & - & $\mathrm{Zn}^{31,32}$ \\
- & $277(\mathrm{~m})$ & $\mathrm{C}-\mathrm{C}-\mathrm{O}$ torsion \\
$346(\mathrm{~m})$ & - & $\mathrm{Zn}-\mathrm{S}^{31,32}$ \\
- & $361(\mathrm{~m})$ & $\mathrm{C}-\mathrm{C}-\mathrm{O}$ bending \\
$420(\mathrm{w})$ & - & $\mathrm{C}-\mathrm{C}-\mathrm{O}$ bending \\
- & $534(\mathrm{~s})$ & $\mathrm{C}-\mathrm{O}$ wagging \\
- & $580(\mathrm{~s})$ & $\mathrm{C}-\mathrm{O}$ rocking \\
$610(\mathrm{br}, \mathrm{w})$ & - & $\mathrm{C}-\mathrm{O}$ bending \\
$848(\mathrm{br}, \mathrm{w})$ & $842(\mathrm{~s})$ & $\mathrm{C}-\mathrm{C}$ stretching \\
$989(\mathrm{~s})$ & $931(\mathrm{~s})$ & $\mathrm{CH}$ rocking \\
$1040(\mathrm{w})$ & $1060(\mathrm{~m})$ & $\mathrm{C}-\mathrm{O}$ stretching \\
$1134(\mathrm{br}, \mathrm{w})$ & $1140(\mathrm{~m})$ & $\mathrm{C}-\mathrm{C}$ stretching \\
- & $1230(\mathrm{~m})$ & $\mathrm{CH} \mathrm{H}_{2}$ twisting \\
$1280(\mathrm{br}, \mathrm{w})$ & $1279(\mathrm{~s})$ & $\mathrm{CH} \mathrm{H}_{2}$ wagging \\
- & $1394(\mathrm{~s})$ & $\mathrm{C}-\mathrm{O}-\mathrm{H}$ bending \\
$1470(\mathrm{w})$ & $1480(\mathrm{~m})$ & $\mathrm{CH}$ bending \\
- & $2832(\mathrm{br}, \mathrm{w})$ & $\mathrm{C}-\mathrm{H}_{\text {stretching, }}$ \\
& & anti-symmetric \\
- & $2884(\mathrm{w})$ & $\mathrm{C}-\mathrm{H}$ stretching, \\
\end{tabular}

$\mathrm{br}=$ broad, $\mathrm{w}=$ weak, $\mathrm{m}=$ medium, $\mathrm{s}=$ strong

data obtained from ZnS:Mn- PEO-35,000 and uncoordinated PEO-35,000 molecules. The peaks were assigned by comparison with those of ethylene glycol ${ }^{32}$ and lithium ion coordinated PEO molecules reported in the literature. ${ }^{33}$ In the spectra, all the $\mathrm{ZnS}: \mathrm{Mn}-\mathrm{PEO}$ nanocrystals did not show the peaks at around $2,800 \sim 3,500 \mathrm{~cm}^{-1}$ which correspond to $\mathrm{O}-\mathrm{H}$ stretching in ethylene glycol. However, it is hard to say that the terminal $\mathrm{OH}$ groups in the PEO capping ligands were deprotonated during the formation of $\mathrm{ZnS}: \mathrm{Mn}-\mathrm{PEO}$ nanocrystals since the preparation condition for the nanocrystals was almost neutral, without using any base. The down shifts of the $\mathrm{C}-\mathrm{O}$ stretching peaks of $\mathrm{ZnS}$ :Mn-PEO $\left(1040 \mathrm{~cm}^{-1}\right)$ from that of uncoordinated PEO $\left(1060 \mathrm{~cm}^{-1}\right)$ indicate that the oxygen atoms in the PEO chains are mostly coordinated to the zinc ions on the surface of the $\mathrm{ZnS}: \mathrm{Mn}$ nanocrystals. In the Raman spectrum, peaks at $346 \mathrm{~cm}^{-1}$ and $261 \mathrm{~cm}^{-1}$ were assigned as surface phonon modes of the $\mathrm{ZnS}$ nanocrystal lattice. Theoretical $\mathrm{ZnS}$ nanocrystal surface phonon peaks are reported as $354 \mathrm{~cm}^{-1}$ and $222 \mathrm{~cm}^{-1}$ in the literature. $^{34}$ They are also close to the Raman peaks appearing in ZnS:Mn-MAA (mercaptoacetic acid) nanocrystal, which were assigned by a DFT calculation. ${ }^{18}$

Acknowledgments. This research was supported by the GRRC program of Gyonggi Province, [GRRC-Dankook-2010B02].

\section{References}

1. Alivisatos, P. J. Phys. Chem. 1996, 100, 13226.

2. Goldman, E. R.; Balighian, H.; Mattoussi, M. K.; Mauro, J. M.; Tran, P. T.; Anderson, G. P. J. Am. Chem. Soc. 2002, 124, 6378

3. Milliron, D. J.; Alivisatos, A. P.; Pitois, C.; Edder, C.; Frechet, J. M. J. Adv. Mater. 2003, 15, 58.

4. Jaiswal, J. K.; Mattoussi, H.; Mauro, J. M.; Simon, S. M. Nature Biotechnol. 2002, 21, 47.

5. Heath, J. R. Acc. Chem. Res. 1999, 32.

6. Hoffman, A. J.; Mills, G.; Yee, H.; Hoffmann, M. R. J. Chem. Phys. 1992, 96, 5546.

7. Kanemoto, M.; Shiragami, T.; Pac, C.; Yanagida, S. J. Phys. Chem. 1992, 96, 3521.

8. Hwang, J. M.; Oh, M. O.; Kim, I.; Lee, J. K.; Ha, C.-S. Curr. Appl. Phys. 2005, 5, 31 .

9. Yu, S. H.; Wu, Y. S.; Yang, J. Chem. Mater. 1998, 9, 2312.

10. Mattousi, H.; Mauro, J. M.; Goldman, E. R.; Anderson, G. P.; Sundar, V. C.; Mikulec, F. V.; Bawendi, M. G. J. Am. Chem. Soc. 2000, 122,12142 .

11. Chan, W. C. W.; Nie, S. Science 1998, 281, 2016.

12. Alivisatos, P. Science 1996, 271, 933.

13. Gerion, D.; Pinaud, F.; Williams, S. C.; Parak, W. J.; Zanchet, D.; Weiss, S.; Alivisatos, A. P. J. Phys. Chem. B 2001, 195, 8861.

14. Chen, C. C.; Yet, C. P.; Wang, H. N.; Chao, C. Y. Langmuir 1999, 15,6845 .

15. Mitchell, G. P.; Mirkin, C. A.; Letsinger, R. L. J. Am. Chem. Soc. 1999, 121,8122 .

16. Hwang, C.-S.; Lee, N. R.; Kim, Y. A.; Park, Y. B. Bull. Korean Chem. Soc. 2006, 27, 1809.

17. Lee, J. H.; Kim, Y. A.; Kim, K. M.; Huh, Y. D.; Hyun, J. W.; Kim, H. S.; Noh, S. J.; Hwang, C.-S. Bull. Korean Chem. Soc. 2007, 28, 1091

18. Kim, J. E.; Hwang, C.-S.; Yoon, S. W. Bull. Korean Chem. Soc. 2008, 29, 1247.

19. Kahovec, J.; Fox, R. B.; Hatada, K. Pure Appl. Chem. 2002, 74, 1921.

20. Lee, J.; Kim, J.; Park, E.; Jo, S.; Song, R. Phys. Chem. Chem. Phys. 2008, 10, 1739 .

21. Kho, R.; Nguyen, L.; Torres-Martinez, C. L.; Mehra, R. K. Biochem. Biophys. Res. Commun. 2000, 272, 29.

22. Yi, G.; Sun, B.; Yang, F.; Chen, D. J. Mater. Chem. 2001, 11, 2928.

23. Tata, M.; Banerjee, S.; John, V. T.; Waguespack, Y.; Mcpherson, G. Coll. Surf. A Phys. Chem. and Eng. Asp. 1997, 127, 39.

24. Zhuang, J.; Zhang, X.; Wang, G.; Li, D.; Yang, W.; Li, T. J. Mater. Chem. 2003, 13, 1853.

25. Bhargava, R. N.; Gallagher, D.; Hong, X.; Nurmikko, A. Phys. Rev. Lett. 1994, 72, 416.

26. Williams, A. T. R.; Winfield, S. A.; Miller, J. N. Analyst. 1983, 108, 1067.

27. Melhuish, W. H. J. Phys. Chem. 1961, 65, 229.

28. Kumbhokjar, N.; Mahamuni, S.; Leppert, V.; Risbud, S. H. Nanostruc. Mater. 1998, 10, 117.

29. Yoo, D. J.; Chung, S. R.; Oh, I. S. J. Emb. Trans. 2002, 17, 137.

30. Yongamalar, R.; Srinivadan, R.; Vinu, A.; Ariga, K.; Bose, A. C. Solid State Commun. 2009, 149, 1919.

31. International Union of Crystallography in International Tables for X-ray Crystallography, Part III; Netherlands: Dordrecht, 1985; p 318.

32. Krishnan, K.; Krishnan, R. S. Proc. Ind. Acad. Sci. A 1966, L14, 111.

33. Caruso, T.; Capoleoni, S.; Cazzanelli, E.; Agostino, R. G.; Villano, P.; Passerini, S. Ionics 2002, 8, 36.

34. Bdulkhadar, M.; Thomas, B. Nano Struc. Mater. 1995, 5, 289. 